\title{
Solvation structure and dynamics of the dimethylammonium cation diluted in liquid water: A molecular dynamics approach
}

Cite as: J. Chem. Phys. 152, 234501 (2020); https://doi.org/10.1063/5.0004204

Submitted: 09 February 2020 . Accepted: 29 May 2020 . Published Online: 15 June 2020

Ioannis Skarmoutsos (D), and Elvira Guardia
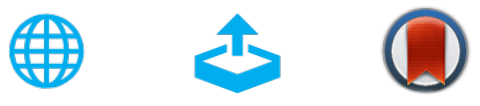

\section{ARTICLES YOU MAY BE INTERESTED IN}

Theory of ion aggregation and gelation in super-concentrated electrolytes

The Journal of Chemical Physics 152, 234506 (2020); https://doi.org/10.1063/5.0006197

On the microscopic origins of relaxation processes in aqueous peptide solutions undergoing a glass transition

The Journal of Chemical Physics 152, 234503 (2020); https://doi.org/10.1063/5.0010312

Using principal component analysis for neural network high-dimensional potential energy surface

The Journal of Chemical Physics 152, 234103 (2020); https://doi.org/10.1063/5.0009264

\section{Lock-in Amplifiers up to $600 \mathrm{MHz}$}
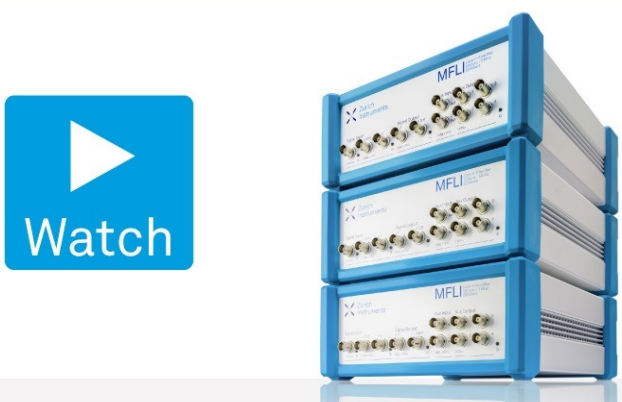


\title{
Solvation structure and dynamics of the dimethylammonium cation diluted in liquid water: A molecular dynamics approach
}

\author{
Cite as: J. Chem. Phys. 152, 234501 (2020); doi: 10.1063/5.0004204 \\ Submitted: 9 February 2020 - Accepted: 29 May 2020 • \\ Published Online: 15 June 2020
}

loannis Skarmoutsos ${ }^{1,2, a)}$ (D) and Elvira Guardia ${ }^{2, a)}$

\begin{abstract}
AFFILIATIONS
${ }^{1}$ Theoretical and Physical Chemistry Institute, National Hellenic Research Foundation, Vass. Constantinou 48, GR-116 35 Athens, Greece

²Departament de Física, Universitat Politècnica de Catalunya, Campus Nord-Edifici B4-B5, Jordi Girona 1-3, Barcelona E 08034, Spain
\end{abstract}

a) Authors to whom correspondence should be addressed: iskarmoutsos@hotmail.com and elvira.guardia@upc.edu

\begin{abstract}
Classical molecular dynamics simulation techniques were employed to investigate the local solvation structure and related dynamics of the dimethylammonium cation diluted in liquid water at ambient conditions. The translational and orientational order around the dimethylammonium cation was investigated in terms of the corresponding radial and angular distribution functions. The results obtained revealed that the first solvation shell of the dimethylammonium consists mainly of two and, less frequently, three water molecules. The two nearest water neighbors form hydrogen bonds with the ammonium hydrogen atoms of the cation, whereas the third neighbor interacts with the methyl hydrogen atoms as well. The distribution of the trigonal order parameter exhibits a bimodal behavior, signifying the existence of local orientational heterogeneities in the solvation shell of the dimethylammonium cation. The calculated continuous and intermittent residence and hydrogen bond lifetimes for the cation-water pairs have also been found to be longer in comparison with the water-water ones. The very similar self-diffusion coefficients of the dimethylammonium cation and the water molecules in the bulk dilute solution indicate that the translational motions of the cation are mainly controlled by the translational mobility of the surrounding water molecules.
\end{abstract}

Published under license by AIP Publishing. https://doi.org/10.1063/5.0004204

\section{INTRODUCTION}

Solvation mechanisms and related dynamics in aqueous electrolytes are complex phenomena, which are of fundamental importance for a deeper understanding of their physicochemical properties and applications. ${ }^{1-17}$ The nature of the intermolecular interactions and the related solvation mechanisms in aqueous electrolytes depends on the type of the salts and the salt concentration. ${ }^{18-20}$ Moreover, the interplay between self and collective dynamics in aqueous electrolyte solutions is still unclear, and it is a topic of active research.

Dilute salt aqueous solutions are often used as prototypes to investigate the effect of the ion type on hydration effects and, particularly, the structural changes in the hydration shells around the ions in comparison with bulk water. ${ }^{23-27}$ Ions having both hydrophobic and hydrophilic groups are extremely interesting prototypes to study the local structure of water in their hydration shells and effects induced by their specific orientational ordering around the cations. In this context, the dimethylammonium cation $\left(\mathrm{DMA}^{+}\right)^{28}$ is an excellent example to study ion hydration at dilute conditions since it contains two hydrophobic groups $\left(-\mathrm{CH}_{3}\right)$ and two hydrogen atoms, which can interact more strongly with the water molecules. Previous studies of one of the authors have revealed that when $\mathrm{DMA}^{+}$is used as a counter cation in anionic metal-organic frameworks, it acts as a nucleation site for water, playing in this way a very important role on water adsorption in this type of nanoporous materials. ${ }^{29}$ The results of that study have indeed revealed the existence of strong interactions of $\mathrm{DMA}^{+}$with water under confinement, and it would be extremely interesting to compare the behavior of the 
$\mathrm{DMA}^{+}$cations and their interactions with water at bulk liquid conditions.

An additional motivation for us to study the solvation structure and related dynamics of this specific cation in water is the fact that a direct comparison can be made with previous studies of one of the authors, who studied the hydration structure and dynamics of the tetramethylammonium cation $\left(\mathrm{TMA}^{+}\right) .{ }^{30} \mathrm{TMA}^{+}$has been used as an ionic model to study hydrophobic behavior in water solutions, and it would be interesting to investigate the changes in the cation solvation structure and related dynamics when two methyl groups are substituted by two hydrogen atoms in the case of $\mathrm{DMA}^{+}$. Very recent $a b$ initio molecular dynamics (MD) studies have also revealed important information regarding the hydration structure of the ammonium cation. ${ }^{31}$ In comparison with ammonium and $\mathrm{TMA}^{+}, \mathrm{DMA}^{+}$is not entirely hydrophobic or hydrophilic since it contains both hydrophobic and hydrophilic groups. A comparison of the structural modifications induced by the substitution of two hydrophobic groups by hydrogen atoms might be used as a springboard toward a better understanding of the solvation phenomena in aqueous electrolytes, particularly related to hydrophobic and hydrophilic hydration effects. Further understanding the mechanisms of these hydration effects could therefore be crucial in the rational design of electrolytes for chemical and environmental applications.

\section{COMPUTATIONAL METHODS AND DETAILS}

In the present study, the solvation structure of $\mathrm{DMA}^{+}$at infinite dilution in pure ambient water $(\mathrm{T}=298.15 \mathrm{~K}$ and $\mathrm{P}=1 \mathrm{bar})$ has been investigated via classical molecular dynamics (MD) simulations. Inside a cubic simulation box, one $\mathrm{DMA}^{+}$cation was placed among 499 water molecules. The initial configuration of the simulated system was prepared by using the Packmol software, ${ }^{32}$ and then, the system was equilibrated by performing an initial $5 \mathrm{~ns} \mathrm{MD}$ simulation at the isothermal-isobaric (NPT) ensemble. The density of the equilibrated system was then calculated by a subsequent 2 ns production NPT-MD run. Using the box dimension corresponding to the calculated density of the system at 1 bar, the system was then re-equilibrated at the canonical (NVT) ensemble for $10 \mathrm{~ns}$, and finally, the system properties under equilibrium were evaluated in a subsequent 5 ns NVT-MD production run. The force field employed in the simulations for the $\mathrm{DMA}^{+}$cation was developed in our previous study of water adsorption in the Y-shp-MOF-5 rare-earthbased metal-organic framework. ${ }^{29}$ The intermolecular interactions in this model are represented as pairwise additive with site-site 126 Lennard-Jones (LJ) plus Coulomb interactions. The $\mathrm{DMA}^{+}$cation was kept rigid during the simulations. The intramolecular coordinates, charges, and LJ parameters corresponding to each type of atoms of the $\mathrm{DMA}^{+}$cation are presented in Table I. The development of potential models for water has been a topic of constant interest for the research community over the last few decades, and a very large variety of force fields have been proposed to describe the thermodynamic, structural, transport, and dynamic properties of water in a wide range of thermodynamic conditions. ${ }^{33,34}$ To be consistent with our previous work, ${ }^{29}$ we selected the rigid Simple Point Charge Extended (SPC/E) potential model ${ }^{35}$ among other force fields to describe water. This three-site potential model has been successfully used in previous studies of liquid and supercritical water as well as in the case of several aqueous mixtures. ${ }^{36-39}$ This model was selected in our previous study of confined $\mathrm{DMA}^{+}$aqueous solutions since it presented the advantage to consider atomic charges for the hydrogen and oxygen atoms very similar to the ones calculated for the coordinated water molecules in the anionic cluster of Y-shp-MOF-5, calculated from our density functional theory (DFT) calculations. ${ }^{29}$ Therefore, in order to be able to directly compare the behavior of $\mathrm{DMA}^{+}$in the bulk solution and under confinement, we used exactly the same force fields as in our previous study.

The equations of motion were integrated using a leapfrogtype Verlet algorithm ${ }^{40}$ with an integration time step of 1 fs. The temperature has been fixed to $298.15 \mathrm{~K}$ and the pressure to 1 bar by coupling the system to a Nose-Hoover thermostat ${ }^{41}$ and barostat $^{42}$ with a relaxation time of $0.5 \mathrm{ps}$, respectively. The rigid body equations of motion for the $\mathrm{DMA}^{+}$cation and the water molecules were expressed in the quaternion formalism. ${ }^{40}$ A cutoff radius of $12.0 \AA$ has been applied for all Lennard-Jones interactions, and long-range corrections have also been taken into account. To account for the long-range electrostatic interactions, the standard Ewald summation technique has been used. ${ }^{40}$ The

TABLE I. Intramolecular coordinates, LJ parameters, and charges corresponding to each atom type of the force field employed in the MD simulations to model the $\mathrm{DMA}^{+}$cation.

\begin{tabular}{lcccrrr}
\hline \hline Atom type & $\mathrm{x}(\AA)$ & $\mathrm{y}(\AA)$ & $\mathrm{z}(\AA)$ & $\sigma(\AA)$ & $\varepsilon(\mathrm{K})$ & $\mathrm{q}(|\mathrm{e}|)$ \\
\hline $\mathrm{C}_{\mathrm{M}}$ & 1.256416 & -0.277080 & 0.000001 & 3.50 & 15.0967 & -0.227 \\
$\mathrm{H}_{\mathrm{M}}$ & 1.254139 & -0.898056 & 0.894676 & 2.50 & 7.5483 & 0.154 \\
$\mathrm{H}_{\mathrm{M}}$ & 1.254120 & -0.898110 & -0.894639 & 2.50 & 7.5483 & 0.154 \\
$\mathrm{H}_{\mathrm{M}}$ & 2.113924 & 0.394560 & -0.000028 & 2.50 & 7.5483 & 0.154 \\
$\mathrm{C}_{\mathrm{M}}$ & -1.256421 & -0.277080 & 0.000001 & 3.50 & 15.0967 & -0.227 \\
$\mathrm{H}_{\mathrm{M}}$ & -1.254164 & -0.898022 & 0.894700 & 2.50 & 7.5483 & 0.154 \\
$\mathrm{H}_{\mathrm{M}}$ & -2.113920 & 0.394568 & -0.000077 & 2.50 & 7.5483 & 0.154 \\
$\mathrm{H}_{M}$ & -1.254092 & -0.898137 & -0.894618 & 2.50 & 7.5483 & 0.154 \\
$\mathrm{~N}$ & 0.000001 & 0.543762 & 0.000001 & 3.30 & 85.5479 & -0.042 \\
$\mathrm{H}_{\mathrm{N}}$ & 0.000007 & 1.160928 & 0.817276 & $\ldots$ & $\ldots$ & 0.286 \\
$\mathrm{H}_{\mathrm{N}}$ & 0.000005 & 1.160892 & -0.817303 & $\cdots$ & $\cdots$ & 0.286 \\
\hline \hline
\end{tabular}


simulation runs were performed using the DL_POLY simulation code. $^{43}$

\section{RESULTS AND DISCUSSION}

\section{A. Local hydration structure}

The solvation structure of the $\mathrm{DMA}^{+}$cation in ambient liquid water was first investigated in terms of the center of mass-center of mass (c.m.-c.m.) radial distribution functions (RDF) $g(r)$ and the corresponding local coordination number $\mathrm{N}(\mathrm{r})$. The $\mathrm{DMA}^{+}-$ water c.m.-c.m. RDF and the corresponding coordination number are presented as a function of the intermolecular distance in Fig. 1(a). The calculated c.m.-c.m. RDF exhibits a peak at $2.98 \AA$, followed by a minimum at $3.38 \AA$, which identifies the radius of the first solvation shell. The average coordination number corresponding to the radius of the first solvation shell has been found to be 2.4. Our analysis revealed that the majority of the obtained first solvation shell configurations (around $66.12 \%$ of the total configurations) contain two water molecules, and there is a significant fraction (around $27.92 \%$ of the total configurations) of configurations containing three water molecules in the first solvation shell. Significantly lower fractions of configurations containing one and four water molecules in the first solvation shell have also been observed. Therefore, the results obtained clearly reveal the existence of a first solvation shell with a radius of $3.38 \AA$ containing mainly two or three water molecules. Following our previous studies, to obtain more detailed insight about the packing of water molecules around the $\mathrm{DMA}^{+}$cation at short distances, the total c.m.-c.m. RDF was expressed in terms of the sum of the different contributions arising from nearest neighbors around the cation. The c.m.-c.m. RDF can indeed be expressed as a sum of the RDF $g^{n}(r),{ }^{44-46}$ where the index $\mathrm{n}$ corresponds to the $\mathrm{n}$-th nearest neighbor around the reference cation,

$$
g(r)=\sum_{n=1}^{\infty} g^{n}(r)
$$

The contributions to the overall c.m.-c.m. RDF corresponding to the first four nearest neighbors are shown in Fig. 1(b). Here, we can also observe that the sum $\sum_{n=1}^{4} g^{n}(r)$ adequately describes the shape of the $\mathrm{DMA}^{+}$-water c.m.-c.m. RDF up to the first minimum located at $3.38 \AA$. Interestingly, the peak of $g^{3}(r)$ is located in the boundary of the first solvation shell, indicating that the third nearest water neighbor alternates between the first and the second solvation shell of the $\mathrm{DMA}^{+}$cation.

A second peak located at $4.42 \AA$, followed by a clear minimum at $6.08 \AA$, can also be observed in the shape of the calculated $\mathrm{DMA}^{+}$-water c.m.-c.m. RDF in Fig. 1(a). This finding clearly indicates the existence of a second solvation shell with a radius of $6.08 \AA$ and a corresponding coordination number $\mathrm{N}_{\mathrm{c}}=29$. Comparing the local intermolecular structure around the $\mathrm{DMA}^{+}$ cation, we can notice an important difference with the local hydration structure around the $\mathrm{TMA}^{+}$cation, which was investigated in a previous study of one of the authors. ${ }^{30}$ In that study, ${ }^{30}$ it was found that the first solvation shell of $\mathrm{TMA}^{+}$, corresponding to the first minimum of the $\mathrm{N}-\mathrm{O}_{\mathrm{w}} \mathrm{RDF}$, which is very similar to the
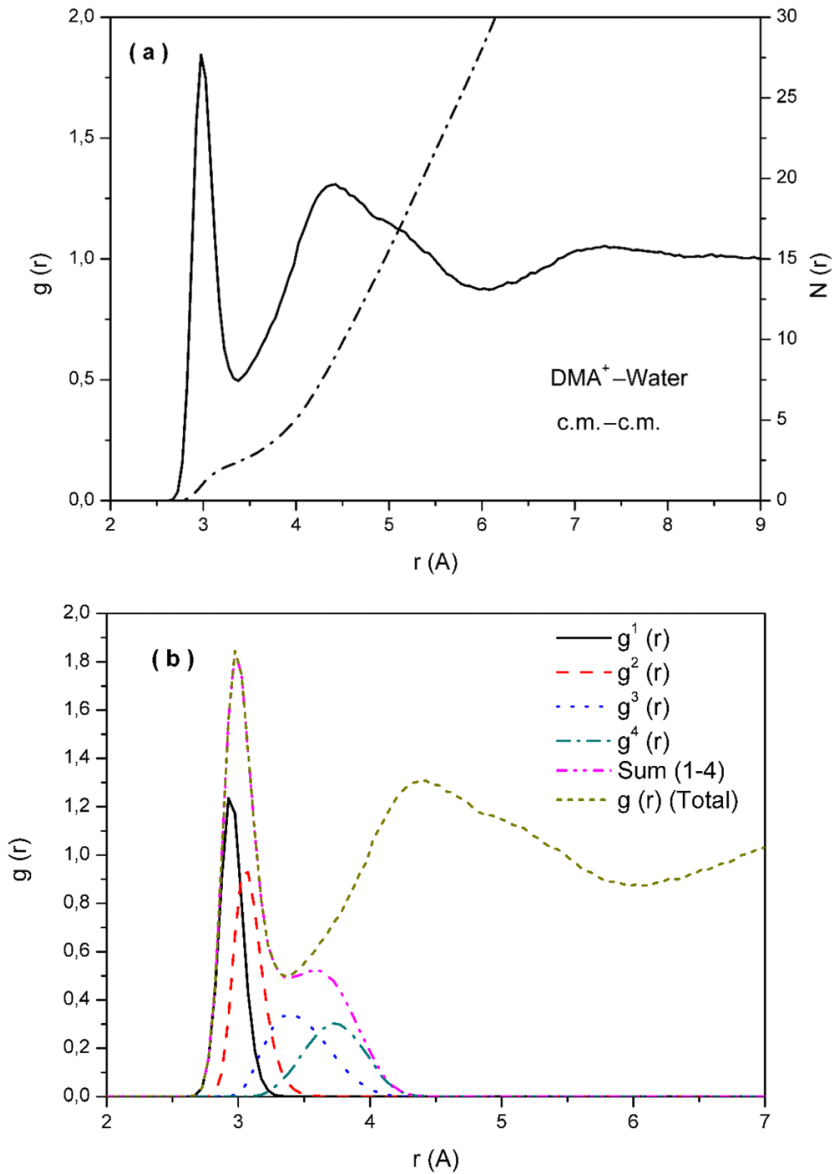

FIG. 1. (a) Calculated DMA ${ }^{+}$-water c.m.-c.m. RDF and the corresponding coordination number. (b) Contributions of the four nearest neighbor RDF $g^{n}(r)$ to the $\mathrm{DMA}^{+}$-water c.m.-c.m. RDF.

c.m.-c.m. one, is located at $6.25 \AA$ and contains 30 water molecules. The existence of two methyl groups and two hydrogen atoms in $\mathrm{DMA}^{+}$leads to the creation of a first solvation shell at short distances, which is absent in the case of $\mathrm{TMA}^{+}$. On the other hand, the location of the second solvation shell is very similar in both cations, as well as the calculated coordination number. ${ }^{30}$ To compare with the hydration structure of the $\mathrm{NH}_{4}{ }^{+}$cation as well, the results of a very recent $a b$ initio Born-Oppenheimer molecular dynamics simulation study ${ }^{31}$ were taken into account. According to the findings of this work, the first solvation shell of $\mathrm{NH}_{4}{ }^{+}$is located at $3.54 \AA$ and contains, on average, 5.2 water molecules. What was also found in this work is that the first solvation shell of $\mathrm{NH}_{4}{ }^{+}$is formed by four tightly bound water molecules and a fifth water molecule that is exchanged between the first and second solvation shells. The structure around $\mathrm{NH}_{4}{ }^{+}$was also found to be much more ordered, and the four nearest water molecules that reside within the first solvation shell are hydrogen bonded with the hydrogen atoms of ammonium, which together form a distinct tetrahedral cage around the ion. All these findings indicate the very 
significant modifications in the hydration structure of these particular ions, caused by the substitution of hydrogen atoms with methyl groups.

The calculated atom-atom RDFs for $\mathrm{DMA}^{+}$-water pairs, presented in Fig. 2, can provide additional insight regarding the appearance of the characteristic first hydration shell in the case of $\mathrm{DMA}^{+}$. As can be observed from Fig. 2, the $\mathrm{H}_{\mathrm{N}}-\mathrm{O}_{\mathrm{w}} \mathrm{RDF}$ exhibits a sharp peak at $1.58 \AA$, followed by a minimum at $2.28 \AA$. This is a very clear indication of the formation of hydrogen bonds between the $\mathrm{DMA}^{+}$cation and its nearest water neighbors, and it is the main reason for the appearance of the first hydration shell in the case of $\mathrm{DMA}^{+}$. On the other hand, the shape of the $\mathrm{H}_{M}-\mathrm{O}_{\mathrm{w}} \mathrm{RDF}$ is very different in comparison with that of the $\mathrm{H}_{\mathrm{N}}-\mathrm{O}_{\mathrm{w}}$ one, exhibiting only a weak shoulder at around $2.9 \AA$, followed by a weak peak at $3.38 \AA$ and a minimum at $3.38 \AA$. This is a clear indication that the hydrogen bonding ( $\mathrm{HB}$ ) interactions between the methyl hydrogen atoms of the cation and the water molecules are not very strong. However, the existence of strong $\mathrm{H}_{\mathrm{N}}-\mathrm{O}_{\mathrm{w}} \mathrm{HB}$ interactions is also reflected on the shape of the $\mathrm{N}-\mathrm{O}_{\mathrm{w}} \mathrm{RDF}$, which is very different in comparison with the one obtained in the case of the $\mathrm{TMA}^{+}$cation. It exhibits a sharp peak at $2.58 \AA$, followed by a first minimum at $3.08 \AA$, whereas the second minimum is located at $5.83 \AA$. The existence of the first minimum in the $\mathrm{N}-\mathrm{O}_{\mathrm{w}}$ $\mathrm{RDF}$ is directly related to the $\mathrm{H}_{\mathrm{N}}-\mathrm{O}_{\mathrm{w}} \mathrm{HB}$ interactions, which are absent in the case of $\mathrm{TMA}^{+}$where only a minimum at $6.25 \AA$ was observed in the previous studies. ${ }^{30}$ All these findings are clear evidences of the strong effect of the $\mathrm{DMA}^{+}$-water $\mathrm{HB}$ interactions on the local hydration structure observed in the dilute $\mathrm{DMA}^{+}$aqueous solution.

Further insight on the interactions of the $\mathrm{DMA}^{+}$cation with its nearest neighbors can be provided by calculating the probability distribution functions $\mathrm{P}(\mathrm{r})$ of the distance between specific atom pairs of the cation and the surrounding water molecules. The probability distribution functions for the distances between the $\mathrm{H}_{\mathrm{N}}$ hydrogen atoms of the $\mathrm{DMA}^{+}$cation and the water oxygen atoms

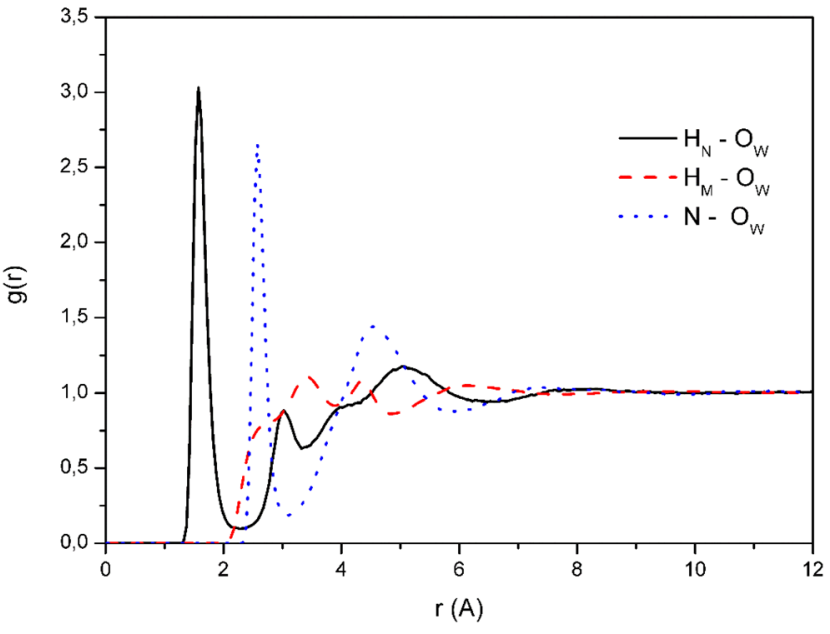

FIG. 2. Calculated atom-atom RDFs for $\mathrm{DMA}^{+}$-water pairs.
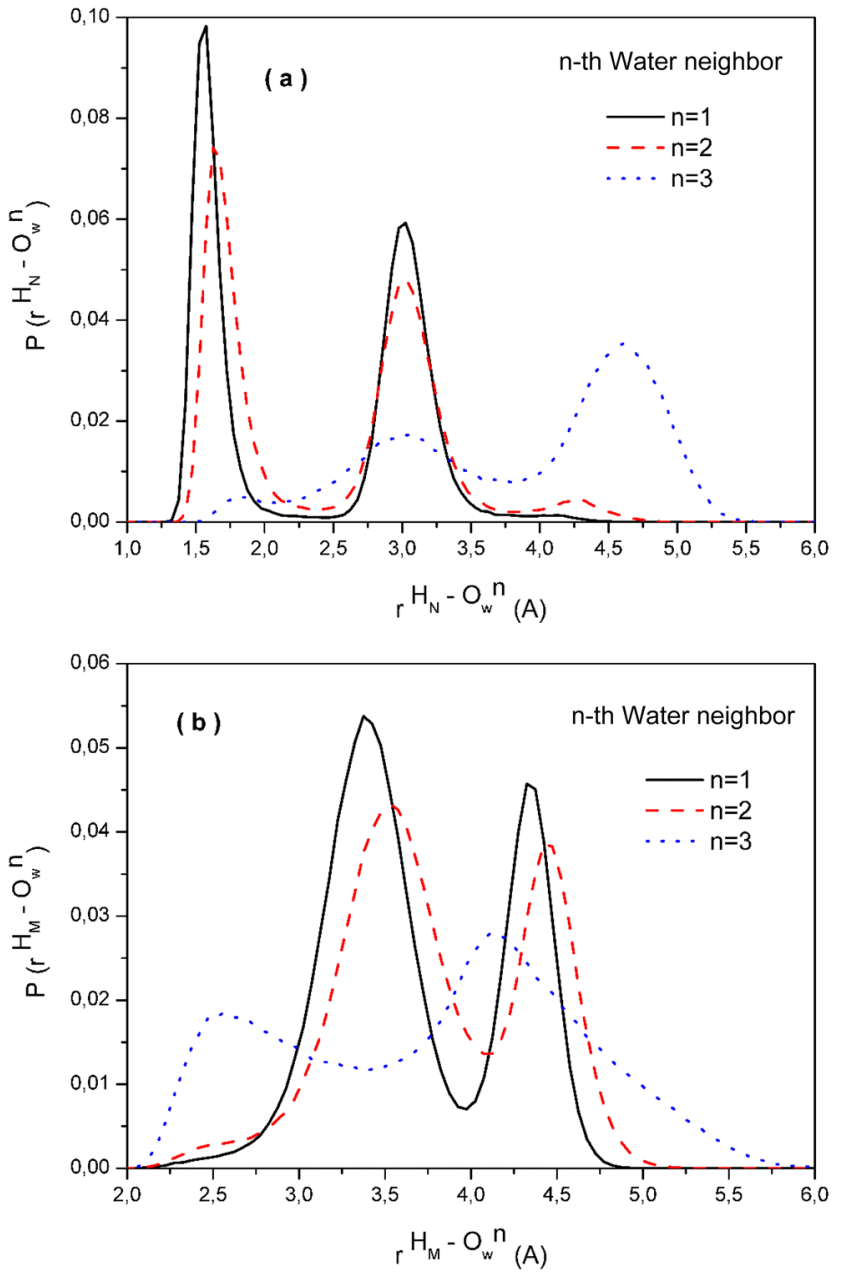

FIG. 3. (a) Calculated probability distribution functions for the distances between the $\mathrm{H}_{\mathrm{N}}$ hydrogen atoms of the $\mathrm{DMA}^{+}$cation and the water oxygen atoms $r^{\mathrm{H}_{N}-\mathrm{O}_{w}}$ of each one of its three nearest water neighbors. (b) Corresponding probability distribution functions for the $r^{H_{M}-O_{w}}$ distances.

$r^{\mathrm{H}_{\mathrm{N}}-\mathrm{O}_{\mathrm{w}}}$ of each one of its three nearest water neighbors are presented in Fig. 3(a). Two intense peaks located at $1.58 \AA$ and $1.63 \AA$ are observed in the distribution functions corresponding to the two nearest water neighbors, followed by a second lower peak at about $3.03 \AA$ A. On the other hand, in the case of the third water neighbor, a very weak shoulder is observed at $1.83 \AA$, followed by two peaks at $3.08 \AA$ and $4.63 \AA$, respectively. These findings clearly indicate that the $\mathrm{DMA}^{+}$cation forms hydrogen bonds with its nearest two water molecules. The probability distribution functions for the distances between the $\mathrm{H}_{\mathrm{M}}$ methyl hydrogen atoms of the $\mathrm{DMA}^{+}$cation and the water oxygen atoms $r^{\mathrm{H}_{\mathrm{M}}-\mathrm{O}_{\mathrm{w}}}$ are also presented in Fig. 3(b). From this figure, it can be clearly observed that the third nearest water neighbor to the $\mathrm{DMA}^{+}$cation prefers to interact with the methyl hydrogen atoms. This is clearly indicated by the appearance of a first peak observed at $2.58 \AA$, which is absent in the case of the 
distance distributions corresponding to the first and second water neighbors. The third nearest water neighbor also exhibits some weaker short-range interactions with the $\mathrm{H}_{\mathrm{N}}$ atoms, reflected in the lower intensity peaks at short range depicted in Fig. 3(a). However, the highest intensity peak is located at $4.63 \AA$, further indicating that the interactions of the third nearest water neighbor with the $\mathrm{H}_{\mathrm{N}}$ atoms are weak. All these findings clearly indicate that the two nearest water neighbors of the $\mathrm{DMA}^{+}$cation approach the two $\mathrm{H}_{\mathrm{N}}$ atoms of the cation and form hydrogen bonds with them, whereas the third water neighbor interacts mainly with the methyl groups of the cation.

The estimation of the number of $\mathrm{H}_{\mathrm{N}} \cdots \mathrm{O}_{\mathrm{w}}$ hydrogen bonds formed between the $\mathrm{DMA}^{+}$cations and the water molecules, with the $-\mathrm{NH}_{2}$ group of the cation acting as a hydrogen bond donor, was performed using geometric criteria defined in our previous studies of confined $\mathrm{DMA}^{+}$aqueous solutions. ${ }^{29}$ According to the employed geometric $\mathrm{HB}$ criterion, a hydrogen bond between $\mathrm{DMA}^{+}$and $\mathrm{H}_{2} \mathrm{O}$ exists if the interatomic distances are defined as follows: $d(N \ldots O)$ $\leq 3.05 \AA$ and $\mathrm{d}\left(\mathrm{H}_{\mathrm{N}} \ldots \mathrm{O}\right) \leq 2.25 \AA$, and the donor-acceptor angle is such as $\mathrm{H}_{\mathrm{N}}-\mathrm{N} \ldots \mathrm{O} \leq 30^{\circ}$. The calculated fractions of $\mathrm{DMA}^{+}$acting as $\mathrm{HB}$ donors and forming from 0 to 3 hydrogen bonds with water were thus estimated. According to our analysis, in about $90 \%$ of the simulated configurations, the cation forms two hydrogen bonds, whereas in about $9.7 \%$ of them, it forms just one bond. The fractions of the configurations where the cation forms zero or three hydrogen bonds are almost negligible $(0.25 \%$ and $0.05 \%$, respectively). The average number of $\mathrm{DMA}^{+}$-water $\mathrm{H}_{\mathrm{N}} \ldots \mathrm{O}_{\mathrm{w}}$ hydrogen bonds formed per $\mathrm{DMA}^{+}$cation was estimated to be about 1.9 , which is the same as in the case of the simulated confined $\mathrm{DMA}^{+}$aqueous solutions in metal-organic frameworks, as was observed in our previous studies.

The local orientational structure around the $\mathrm{DMA}^{+}$cation has also been investigated in terms of angular probability distribution functions $\mathrm{P}(\cos \theta)$. In Fig. 4(a), the distribution of the cosine of the angle formed between the nitrogen atom of the cation and the oxygens of the two nearest water neighbors $\left(\mathrm{O}^{1}-\mathrm{N}-\mathrm{O}^{2}\right.$ angle $)$ is presented. This particular distribution exhibits a peak at a cosine value of -0.26 corresponding to an angle of about $105^{\circ}$. The dependence of this distribution on the distance of the first and second water neighbors from the c.m. of the cation ( $\mathrm{r}^{1-\text { Cation }}$ and $\mathrm{r}^{2-\text { Cation, }}$ respectively) has been further investigated in Figs. 4(b) and 4(c), where the contour plots of the distributions $\mathrm{P}\left(\cos \theta, \mathrm{r}^{1-\text { Cation }}\right)$ and $\mathrm{P}\left(\cos \theta, \mathrm{r}^{2-\text { Cation }}\right)$ are presented. The shape of these plots is quite similar with the distributions exhibiting the maximum probability values for the first and second neighbor distances of about 2.95 $\AA$ and $3.05 \AA$, respectively. The local orientational structure of the third nearest water neighbor around the cation has also been studied in terms of the angular probability distribution function $\mathrm{P}(\cos \theta)$. The probability distribution functions of the angle formed between the nitrogen atom of the cation and the oxygen atom of the third nearest water neighbor with either the oxygen atom of the first $\left(\mathrm{O}^{1}-\mathrm{N}-\mathrm{O}^{3}\right.$ angle) or the second $\left(\mathrm{O}^{2}-\mathrm{N}-\mathrm{O}^{3}\right.$ angle) nearest water neighbor are presented in Fig. 5(a). The contour plots of the corresponding $\mathrm{P}\left(\cos \theta, \mathrm{r}^{3-\text { Cation }}\right)$ probability functions for each one of the aforementioned angles are also presented in Figs. 5(b) and 5(c) $\left(\mathrm{r}^{3-\text { Cation }}\right.$ is the distance of the third nearest water neighbor from the c.m. of the cation). From all these calculated probability distribution functions, it can be clearly observed that the preferred
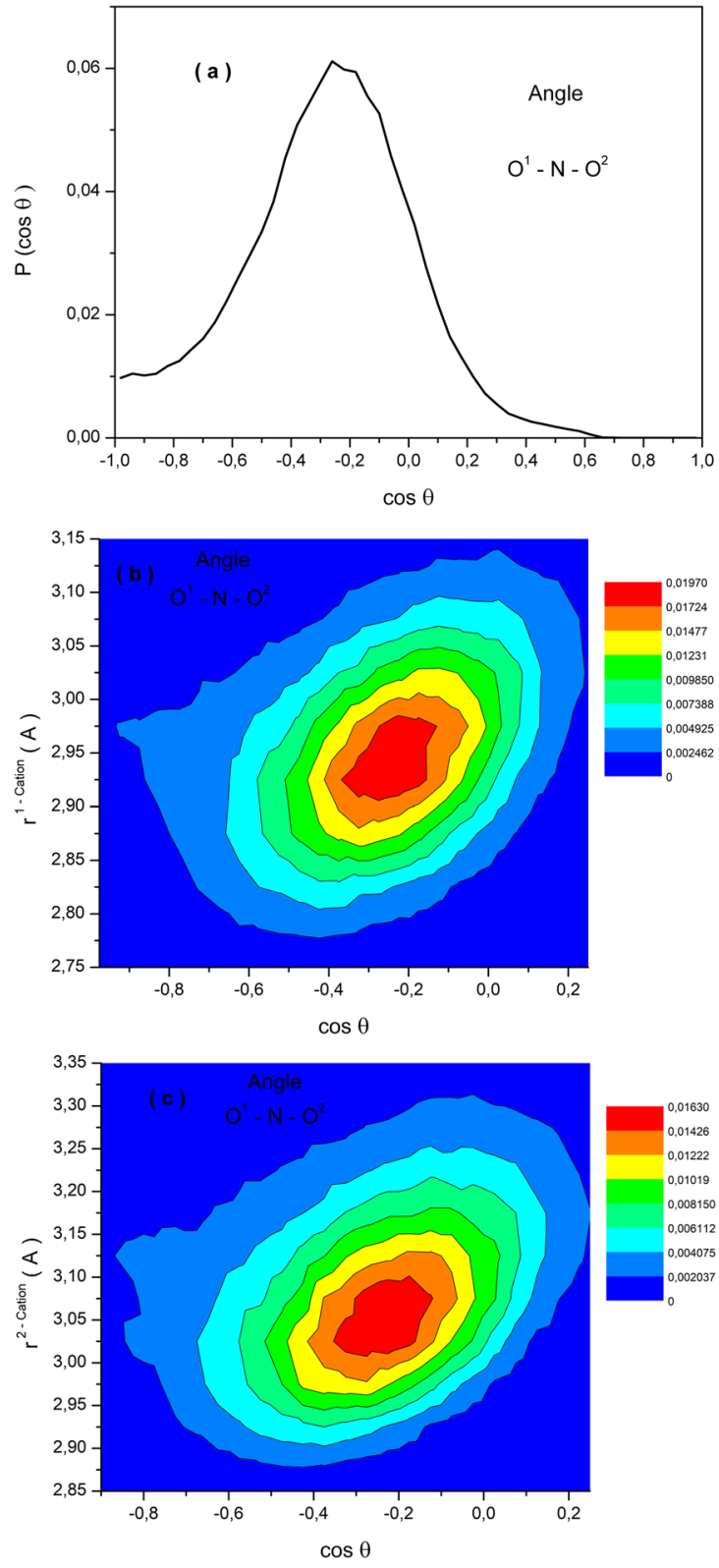

FIG. 4. (a) Calculated cosine distribution for the angle formed between the nitrogen atom of the cation and the oxygens of the two nearest water neighbors $\left(\mathrm{O}^{1}-\mathrm{N}-\mathrm{O}^{2}\right.$ angle). (b) Contour plot of the distribution $\mathrm{P}\left(\cos \theta, \mathrm{r}^{1-\operatorname{cation}}\right)$. (c) Contour plot of the distribution $\mathrm{P}\left(\cos \theta, \mathrm{r}^{2-\text { Cation }}\right)$ for the $\mathrm{O}^{1}-\mathrm{N}-\mathrm{O}^{2}$ angle.

angles are either in the range close to $65^{\circ}$ or higher than $150^{\circ}$, obviously depending on which is the methyl group that interacts with the third nearest water neighbor. These probabilities are maximized for the third water neighbor distances being in the range $3.40 \AA-3.45 \AA$.

The local orientational structure in liquids can, in general, be also investigated in terms of a widerange of orientational 

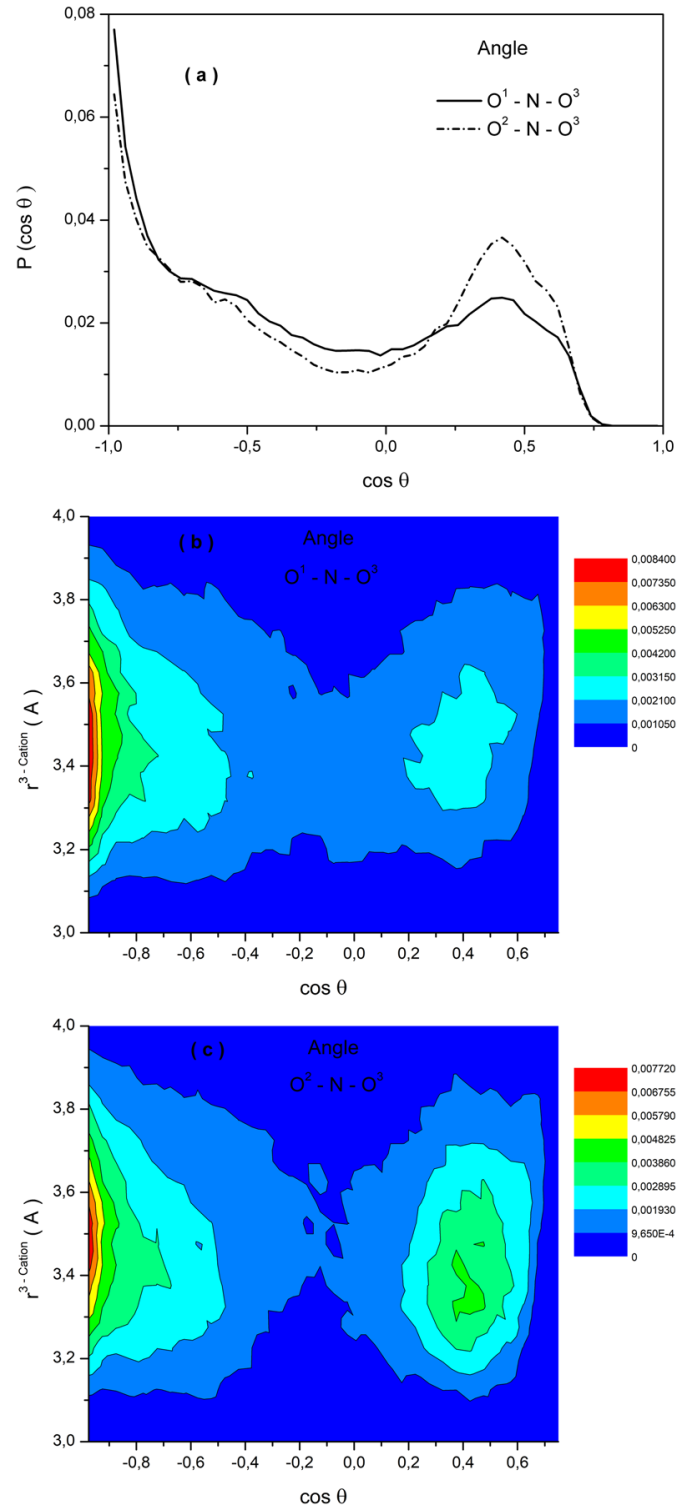

FIG. 5. (a) Calculated cosine distributions for the $\mathrm{O}^{1}-\mathrm{N}-\mathrm{O}^{3}$ and $\mathrm{O}^{2}-\mathrm{N}-\mathrm{O}^{3}$ angles. (b) Contour plot of the distribution $\mathrm{P}\left(\cos \theta, \mathrm{r}^{3-\text { Cation }}\right)$ for the $\mathrm{O}^{1}-\mathrm{N}-\mathrm{O}^{3}$ angle. (c) Contour plot of the distribution $\mathrm{P}\left(\cos \theta, \mathrm{r}^{3-\text { Cation }}\right)$ for the $\mathrm{O}^{2}-\mathrm{N}-\mathrm{O}^{3}$ angle.

structural order parameters. ${ }^{47}$ In this particular case, taking into account the fact that the three nearest water neighbors play an important role in the stabilization of the solvation structure around the cation, we decided to calculate the trigonal order parameter, ${ }^{48,49}$ which is defined in terms of the three nearest neighbors of the reference cation $i$,

$$
q_{3}=1-\left\langle\frac{4}{7} \sum_{j=1}^{2} \sum_{k=j+1}^{3}\left(\cos \phi_{j i k}+\frac{1}{2}\right)^{2}\right\rangle,
$$

and it takes a value of one for the perfectly ordered case and of zero for a random distribution. Here, $\phi_{j i k}$ is the angle formed by the vectors $\vec{r}_{i j}$ and $\vec{r}_{i k}$ connecting the c.m. of the anion i to the c.m. of two of the three nearest water neighbors $\mathrm{j}$ and $\mathrm{k}$. In the perfectly ordered case, the ideal angle $\varphi_{j i k}$ takes the value of $\varphi_{j i k}=120^{\circ}$. The calculated probability distribution for $q_{3}$ is presented in Fig. 6. From Fig. 6, it can be clearly observed that the calculated distribution function is bimodal, exhibiting two peaks, one observed at $q_{3}=0.16$ and the second at $q_{3}=0.74$. Therefore, solvation structures with low and high trigonal symmetries can be observed. This finding is in agreement with the bimodal character of the distributions presented in Figs. 5(b) and 5(c), indicating that there are two preferential configurations for the $\mathrm{O}^{1}-\mathrm{N}-\mathrm{O}^{3}$ and $\mathrm{O}^{2}-\mathrm{N}-\mathrm{O}^{3}$ angles. We have characterized as low- $q_{3}$ solvation structures the ones having $q_{3} \leq 0.46$, which is the value corresponding to the minimum observed in the calculated probability distribution function presented in Fig. 6. The structures having $q_{3}>0.46$ have been characterized as high- $\mathrm{q}_{3}$ solvation structures. Our analysis has revealed that the fractions of the low- and high- $\mathrm{q}_{3}$ solvation structures are about 0.59 and 0.41 , respectively. Our previous studies on $\mathrm{Li}^{+}$solvation in mixtures of organic carbonate electrolytes exhibiting high tetrahedral ordering around the $\mathrm{Li}^{+}$ cation $^{50}$ had revealed a strong effect of the local orientational ordering around $\mathrm{Li}^{+}$on the magnitude of the total local dipole moment of the solvent molecules in the first solvation shell around the cation. For this reason, we decided to further investigate the effect of the local trigonal order of the three nearest water neighbors $(i=1-3)$ of the $\mathrm{DMA}^{+}$cation on their total dipole moment $\vec{M}_{\text {tot }}{ }^{\text {neib } 1-3}=\sum_{i=1}^{3} \vec{\mu}_{i}$. The probability distribution function of the magnitude of the total dipole moment $M_{t o t}{ }^{\text {neib 1-3 }}=\left|\vec{M}_{\text {tot }}{ }^{\text {neib 1-3 }}\right|$ of the three nearest water neighbors of the $\mathrm{DMA}^{+}$cation for low- and high- $\mathrm{q}_{3}$ solvation structures is presented in Fig. 7(a). From this figure, it can be clearly seen that the appearance of a strong local trigonal order around the cation leads to the decrease in the magnitude of the total dipole moment $M_{\text {tot }}{ }^{\text {neib 1-3 }}$. The calculated average values corresponding to the

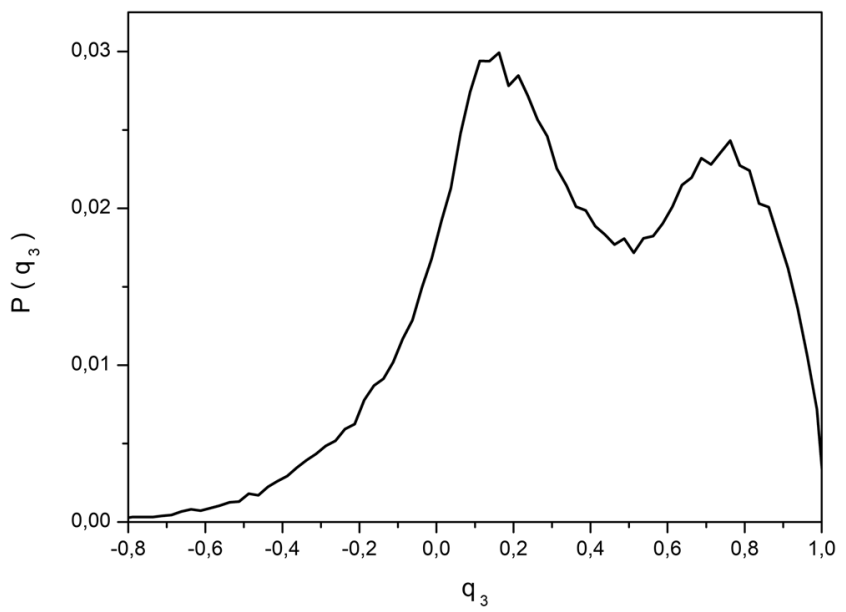

FIG. 6. Calculated probability distribution function for the trigonal order parameter $q_{3}$ of the water molecules around the $\mathrm{DMA}^{+}$cation 

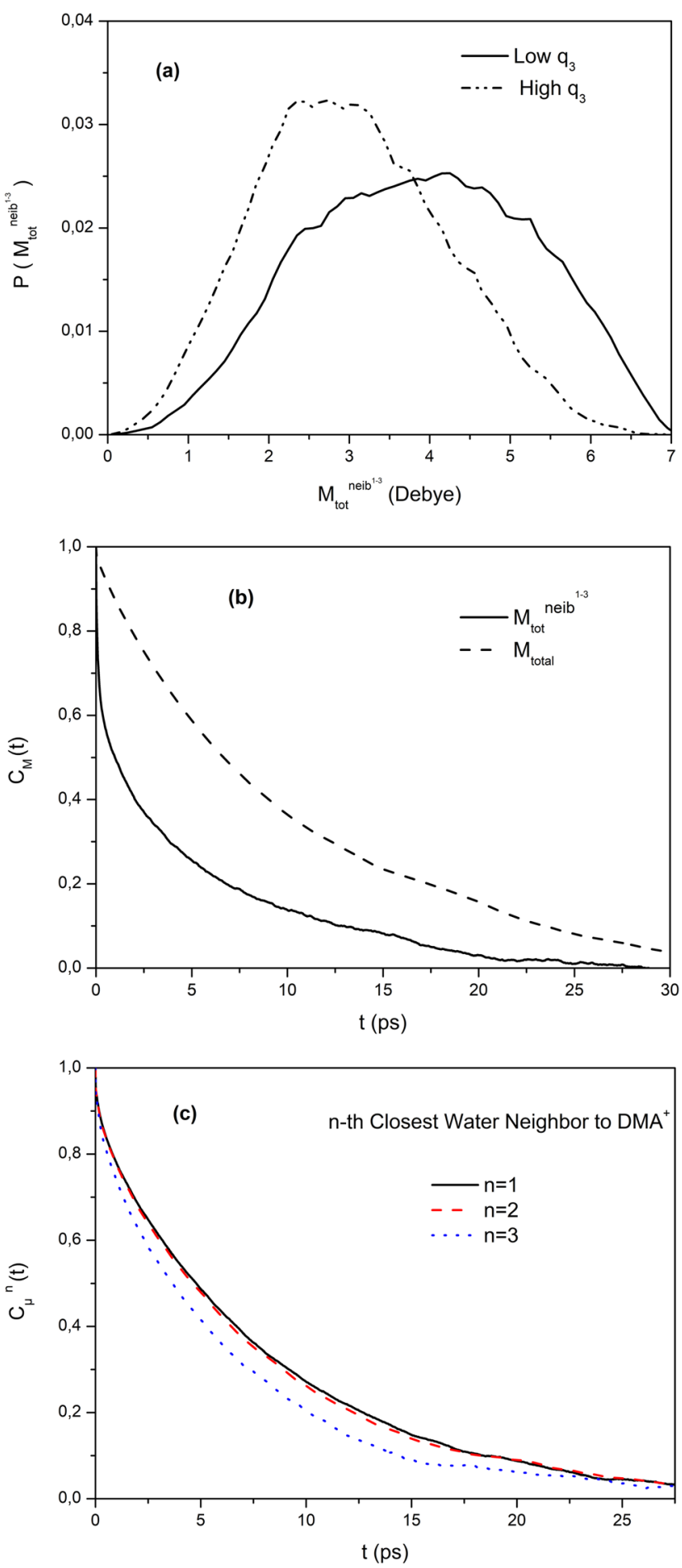

FIG. 7. (a) Probability distribution function of the magnitude of the total dipole moment of the three nearest water neighbors of the $\mathrm{DMA}^{+}$cation, $\vec{M}_{t o t}{ }^{\text {neib }}{ }^{1-3}$, for low- and high- $q_{3}$ solvation structures. (b) Calculated TCFs corresponding to $\vec{M}_{\text {tot }}{ }^{\text {neib 1-3 }}$ and the total dipole moment of all the water molecules in the system $\vec{M}_{\text {tot }}$. (c) Reorientational TCFs of the molecular dipole moment vectors of each one of the three nearest water neighbors of the cation at $t=0$. low- and high- $\mathrm{q}_{3}$ solvation structures are $3.88 \mathrm{D}$ and $3.02 \mathrm{D}$, respectively. According to simple vector calculus, the minimum value for the magnitude of the total dipole vector is zero, which is the case where the molecular dipole vectors are aligned in a perfect trigonal order. The maximum value for the magnitude of the total dipole vector is $7.05 \mathrm{D}$, which is three times the magnitude of the dipole moment of the SPC/E water molecule $(2.35 \mathrm{D})$. The existence of a strong trigonal ordering around the cation therefore causes dipole cancellation effects, and the magnitude of the total dipole moment $M_{\text {tot }}{ }^{\text {neib 1-3 }}$ decreases. The local orientational dipolar ordering of the three nearest water neighbors around the cation can also be reflected in the local finite Kirkwood factor,

$$
G_{K}^{\text {local }}=\frac{\left\langle\left(\vec{M}_{\text {tot }}{ }^{\text {neib 1-3 }}\right)^{2}\right\rangle}{\left\langle 3 \vec{\mu}^{2}\right\rangle} .
$$

The local Kirkwood factor provides a measure of the local orientational properties of the water dipole vectors, and how this local orientational structure is reflected on the total dipole vector. The average calculated value of $G_{K}{ }^{\text {local }}$ was 0.86 , where the calculated bulk value of the finite Kirkwood factor $G_{K}=\frac{\left\langle\vec{M}^{2}\right\rangle}{\left\langle N \vec{\mu}^{2}\right\rangle}$, taking into account all the water molecules in the system, was 3.36. The calculated values of $G_{K}{ }^{\text {local }}$ for the low- and high- $\mathrm{q}_{3}$ solvation structures were 1.02 and 0.63 , respectively. This finding further indicates the strong effect of the local orientational order on the local dielectric properties of the environment around the $\mathrm{DMA}^{+}$ cation.

The investigation of the local Kirkwood factor in liquids is very often studied in the literature. ${ }^{46,51,52}$ We should note, for instance, one of our previous works ${ }^{46}$ where we evaluated the significant differences in the local Kirkwood factor in polymorphs of the plastic crystal phase of water, which, of course, are reflected on the significant differences observed in the dielectric constant of these polymorphs. In the case of electrolyte solutions, as also mentioned above, we had also revealed that the local dipolar orientational symmetry of organic solvent molecules around the lithium cations can play an important role in the local dielectric properties of battery electrolytes and is possibly correlated with the local preferential solvation of lithium cations in mixed electrolyte solvents. ${ }^{50}$ All these are clear indications of the importance of the investigation of local dielectric effects in liquids. From an experimental point of view, it is not trivial to measure local dielectric properties, and for this reason, molecular simulation can be a very useful tool. However, there are experimental studies in the literature $^{53}$ where they have revealed the importance of local dielectric effects, particularly in the case of nanocomposite materials. In these experiments, it has been revealed that the local dielectric properties are strongly correlated with the properties of the interface formed between nanoparticles and polymers. The necessity to develop novel experimental techniques to study local dielectric properties has been pointed out.

\section{B. Dynamic properties}

Apart from the static description of the local structure around the $\mathrm{DMA}^{+}$cation, the dynamics of the local solvation structure were 
also investigated. The time correlation function (TCF) of the time dependent total dipole moment of the three nearest water neighbors $\vec{M}_{\text {tot }}{ }^{\text {neib 1-3 }}$ was calculated and compared with the one corresponding to the total dipole moment of all the water molecules in the system $\vec{M}_{t o t}=\sum_{i=1}^{N} \vec{\mu}_{i}$

$$
C_{M}(t)=\frac{\left\langle\vec{M}_{t o t}(0) \cdot \vec{M}_{t o t}(t)\right\rangle}{\left\langle\vec{M}_{t o t}(0)^{2}\right\rangle} .
$$

The relaxation time associated with the time decay of this TCF can be calculated using the following relation:

$$
\tau_{M}=\int_{0}^{\infty} C_{M}(t) \cdot d t
$$

The calculated TCFs corresponding to $\vec{M}_{t o t}{ }^{\text {neib } 1-3}$ and the total dipole moment of all the water molecules in the system $\vec{M}_{t o t}$ are presented in Fig. 7(b), where it can be clearly observed that the TCF of the total dipole moment of the three nearest water neighbors $\vec{M}_{\text {tot }}{ }^{\text {neib 1-3 }}$ decays much faster. This is also reflected on the calculated relaxation times of these TCFs. The values of $\tau_{M}$ obtained for the dynamics of $\vec{M}_{t o t}{ }^{n e i b 1-3}$ and $\vec{M}_{t o t}$ were 3.9 ps and $10.1 \mathrm{ps}$, respectively. This is a clear indication that the reorientational dynamics of the total dipole moment of the three nearest water neighbors of the cation take place at smaller time scales, whereas the value of $\tau_{M}$ obtained for the total dipole moment of all the water molecules of the system is larger and close to the Debye relaxation time of bulk liquid water. ${ }^{5}$

The reorientational dynamics of the molecular dipole moment vectors of each one of the three nearest water neighbors at $t=0$ were also investigated by calculating the corresponding TCF,

$$
\begin{aligned}
& C_{\mu}{ }^{n}(t)=\frac{\left\langle\vec{\mu}_{i}(0) \cdot \vec{\mu}_{i}(t)\right\rangle}{\left\langle\vec{\mu}_{i}(0)^{2}\right\rangle}, \\
& \mathrm{i}=\text { nth nearest neighbour of the cation at } \mathrm{t}=0, \mathrm{n}=1,3 .
\end{aligned}
$$

The calculated TCFs are presented in Fig. 7(c), where it can be seen that the dipole moment vector of the third nearest water neighbor reorients slightly faster in comparison with the first and second ones, probably due to the fact that its interactions with the cation are slightly weaker. On the other hand, the decays of the $C_{\mu}{ }^{1}(t)$ and $C_{\mu}{ }^{2}(t)$ TCFs corresponding to the first and second nearest neighbors are very similar. These findings are more clearly reflected on the calculated relaxation times of these TCFs, which have been estimated to be $7.5 \mathrm{ps}, 7.4 \mathrm{ps}$, and $6.1 \mathrm{ps}$ for the first, second, and third nearest water neighbors of the cation, respectively. As can be seen, the relaxation times corresponding to the two nearest neighbors are larger than the one corresponding to the third neighbor, possibly due to the fact that they form hydrogen bonds with the $\mathrm{NH}_{2}$ group of the ion. The relaxation times are also significantly higher than the reorientational relaxation time of the total dipole vector $\vec{M}_{t o t}{ }^{\text {neib } 1-3}$, indicating that the cross correlation functions of the dipoles of the nearest neighbors have an important effect on the dynamics of the total dipole and actually speed up these dynamics.
The interaction of the water molecules with the $\mathrm{DMA}^{+}$cation has also been investigated in terms of their c.m.-c.m. pair residence dynamics. According to the literature, the residence TCF inside a solvation shell around a central molecule (or ion) i could be defined as ${ }^{14,55,56}$

$$
C_{r e s}(t)=\frac{\left\langle n_{i j}(0) \cdot n_{i j}(t)\right\rangle_{t^{\star}}}{\left\langle n_{i j}(0)^{2}\right\rangle} .
$$

The corresponding residence time is defined as

$$
\tau_{\text {res }}=\int_{0}^{\infty} C_{r e s}(t) \cdot d t .
$$

The variable $n_{i j}$ takes the value $n_{i j}(t)=1$ if molecule $\mathrm{j}$ is inside the solvation shell of molecule $i$ at times 0 and $t$ and molecule $j$ has not left, in the meantime, the shell for a period longer than $t^{*}$. Otherwise, it takes the value $n_{i j}(t)=0$. Of course, using this definition, the calculation of $C_{r e s}(t)$ depends upon the selection of parameter $\mathrm{t}^{*}$. The two limiting cases arising from this definition are (a) if $t^{*}=0$, which represents the so-called continuous definition, and (b) if $t^{*}=\infty$, which represents the intermittent definition. We have to mention that these two definitions describe very different aspects of residence dynamics since, according to the continuous definition, the exits of molecule $j$ outside the shell of molecule $i$ during the time interval $[0, t]$ are not allowed. On the other hand, in the intermittent case, the persistence of molecule $j$ in the solvation shell of $i$ at time $t$ is investigated, regardless of multiple exits and entrances of this molecule in the shell during the time interval $[0, t]$. In the present work, we have calculated both the continuous and intermittent residence functions $C_{r e s}{ }^{C}(t)$ and $C_{\text {res }}{ }^{I}(t)$ for the first solvation shell of the $\mathrm{DMA}^{+}$cation, which are presented in Fig. 8(a). The calculated continuous and intermittent residence lifetimes $\tau_{\text {res }}{ }^{C}$ and $\tau_{\text {res }}{ }^{I}$ have been found to be $2.6 \mathrm{ps}$ and $12.1 \mathrm{ps}$, respectively.

In a similar way, the dynamics of the $\mathrm{DMA}^{+}$-water hydrogen bonds were also investigated in terms of the corresponding $C_{H B}(t)$ time correlation functions (TCFs), ${ }^{14,55,57}$

$$
C_{H B}(t)=\frac{\left\langle h_{i j}(0) \cdot h_{i j}(t)\right\rangle_{t^{\star}}}{\left\langle h_{i j}(0)^{2}\right\rangle},
$$

while the HB lifetime $\tau_{H B}$ is defined as

$$
\tau_{H B}=\int_{0}^{\infty} C_{H B}(t) \cdot d t .
$$

The variable $h_{i j}$ is such as $h_{i j}(t)=1$ when molecule $\mathrm{j}$ is hydrogen bonded with molecule $i$ at times 0 and $t$, and the corresponding hydrogen bond persists for a period longer than $t^{*}$; otherwise, $h_{i j}(t)=0$. As in the case of residence dynamics, $t^{*}=0$ corresponds to the continuous HB dynamics, and $t^{*}=\infty$ corresponds to the intermittent one. The calculated continuous and intermittent TCFs $C_{H B}{ }^{C}(t)$ and $C_{H B}{ }^{I}(t)$ are presented in Fig. 8(b), and the estimated continuous and intermittent HB lifetimes, $\tau_{H B}{ }^{C}$ and $\tau_{H B}{ }^{I}$, were $1.9 \mathrm{ps}$ and $10.7 \mathrm{ps}$, respectively. These lifetimes are very similar to the 

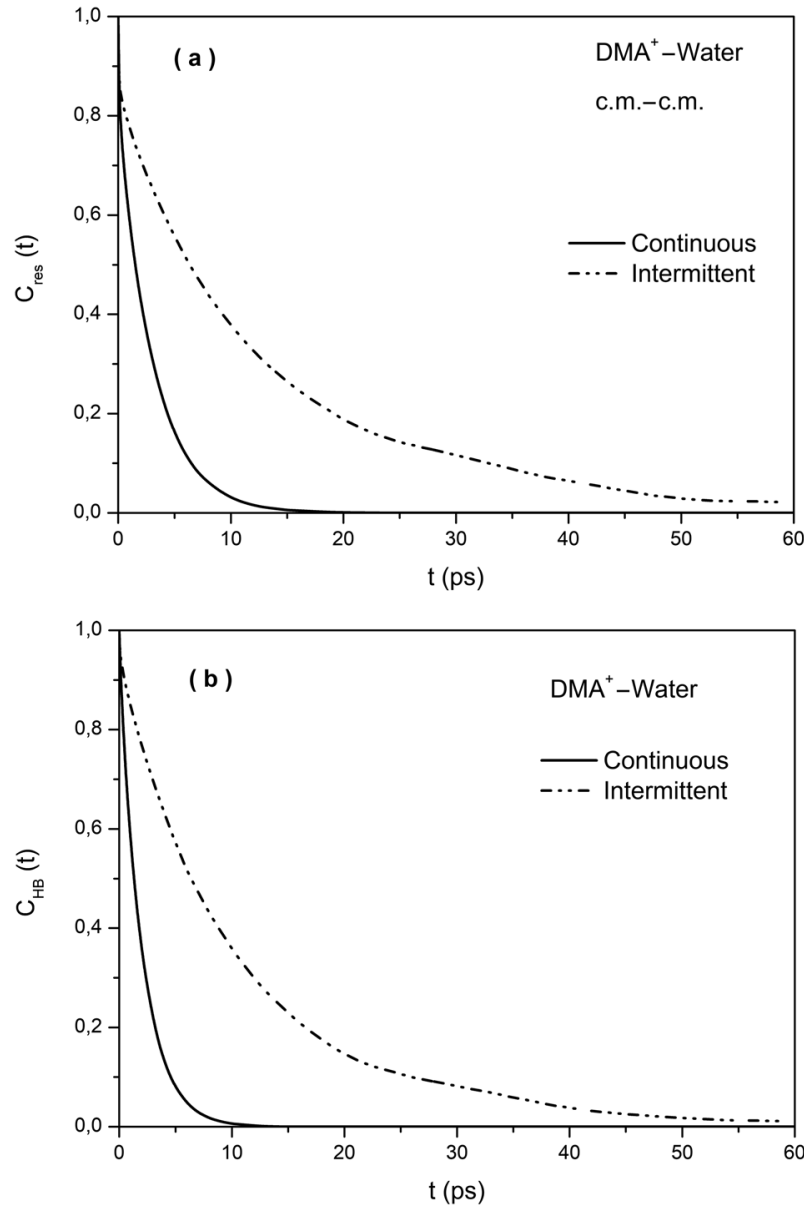

FIG. 8. (a) Calculated continuous and intermittent water residence functions $C_{\text {res }}{ }^{C}(t)$ and $C_{\text {res }}{ }^{I}(t)$ for the first solvation shell of the $\mathrm{DMA}^{+}$cation. (b) Calculated continuous and intermittent TCFs $C_{H B}{ }^{C}(t)$ and $C_{H B}{ }^{I}(t)$ for DMA ${ }^{+}$-water hydrogen bonds.

corresponding residence lifetimes for the first solvation shell of the cation, signifying the strong effect of the $\mathrm{DMA}^{+}$-water $\mathrm{HB}$ interactions on the dynamic reorganization of the water molecules in the first solvation shell of $\mathrm{DMA}^{+}$cation. Note that in our previous studies, ${ }^{29}$ the calculated intermittent HB lifetime $\tau_{H B}{ }^{I}$ for the $\mathrm{DMA}^{+}$-water hydrogen bonds under confinement in the Y-shpMOF-5 porous material was $63.9 \mathrm{ps}$, signifying that $\mathrm{HB}$ dynamics at the bulk solution are significantly faster.

The dynamics of the local orientational structural order have also been investigated in terms of the TCF $C_{q_{3}}(t)$ of the trigonal order parameter $q_{3}$,

$$
C_{q_{3}}(t)=\frac{\left\langle\delta q_{3}(0) \cdot \delta q_{3}(t)\right\rangle}{\left\langle\delta q_{3}(0)^{2}\right\rangle}, \delta q_{3}(t)=q_{3}(t)-\left\langle q_{3}\right\rangle
$$

The corresponding correlation time $\tau_{q_{3}}$ associated with the relaxation of the corresponding orientational order parameter has been calculated using the relation

$$
\tau_{q_{3}}=\int_{0}^{\infty} C_{q_{3}}(t) \cdot d t
$$

The calculated TCF $C_{q_{3}}(t)$ is presented in Fig. 9. From this figure, it can be observed that the time decay of this TCF is very fast, something that is also reflected on the calculated correlation time $\tau_{q_{3}}$ which has the value of 0.17 ps. This finding indicates that the local orientational order around the first solvation shell of the cation changes very rapidly. This finding is also consistent with the findings of our previous work on pure liquid water, ${ }^{58}$ where it was found that the dynamics of the tetrahedral order parameter in liquid water take place at a similar time scale, indicating that small deviations in the local orientational ordering of the nearest neighbors around an ion or a water molecule can cause significant changes in the corresponding order parameters and significantly speed up their dynamics.

The reorientational dynamics of the $\mathrm{DMA}^{+}$cation was also studied in terms of the Legendre reorientational TCFs for specific intramolecular vectors of the cation,

$$
C_{\ell R}(t)=P_{\ell}\left\langle\vec{u}_{i}(0) \cdot \vec{u}_{i}(t)\right\rangle .
$$

The corresponding Legendre reorientational correlation times can be calculated using the following equation:

$$
\tau_{\ell R}=\int_{0}^{\infty} C_{\ell R}(t) \cdot d t .
$$

In Eq. (13), $\vec{u}_{i}$ is a unit bond vector associated with a molecule (or ion) $\mathrm{i}$, and $P_{\ell}$ is a Legendre polynomial of order $\ell$. The calculated first, second, third, and fourth order Legendre reorientational TCFs for the $\mathrm{N}-\mathrm{H}$ and $\mathrm{N}-\mathrm{C}$ vectors of the $\mathrm{DMA}^{+}$ cation are presented in Figs. 10(a) and 10(b), and the estimated

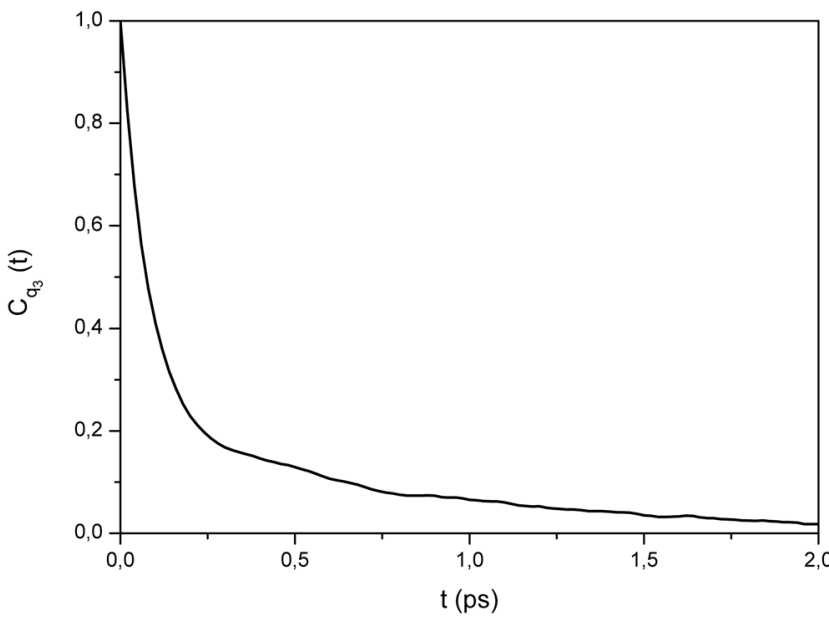

FIG. 9. Calculated TCF $C_{q_{3}}(t)$ corresponding to the trigonal order parameter $q_{3}$ of the water molecules around the $\mathrm{DMA}^{+}$cation. 

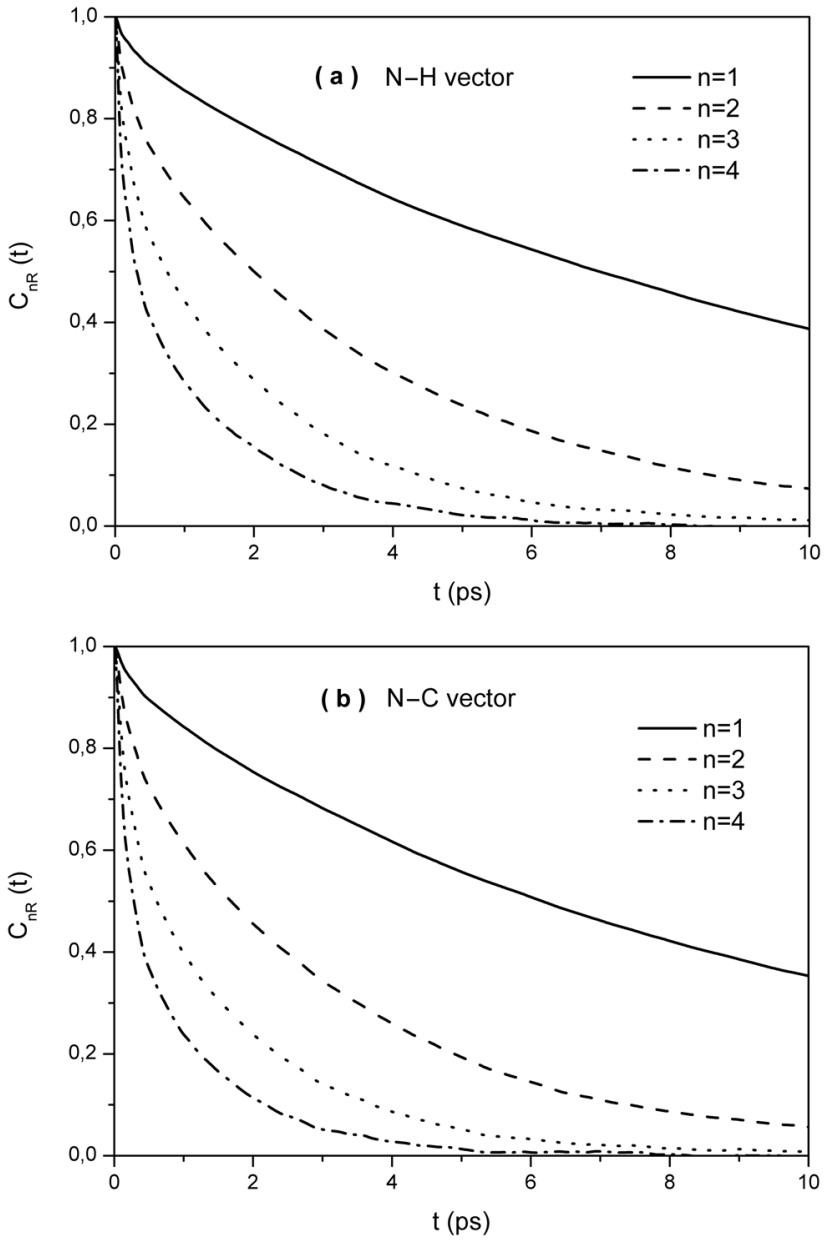

FIG. 10. Calculated first, second, third, and fourth order Legendre reorientational TCFs for the (a) $\mathrm{N}-\mathrm{H}$ and (b) $\mathrm{N}-\mathrm{C}$ vectors of the $\mathrm{DMA}^{+}$cation.

corresponding correlation times are presented in Table II. From these figures, it can be observed that the decay of these reorientational TCFs is significantly faster in comparison with the corresponding dynamics of the cation under confinement, as was revealed in our previous studies. This difference in the reorientational dynamics in the bulk solution is more clearly reflected on the calculated reorientational correlation times. For instance, using as a model fitting function, a sum of three exponential decay functions, the estimated first order Legendre reorientational time $\tau_{1 R}$ of the $\mathrm{N}-\mathrm{H}$ vector of the cation in the bulk solution and under confinement in the Y-shp-MOF-5 porous material ${ }^{29}$ was 10.8 ps and $83.8 \mathrm{ps}$, respectively. There is also a very clear deviation of the calculated Legendre reorientational correlation times from the trends expected when using the Debye diffusion model of reorientational relaxation $^{59-61}\left(\tau_{1 R}=3 \cdot \tau_{2 R}, \tau_{1 R}=6 \cdot \tau_{3 R}\right.$, and $\tau_{1 R}=$ $\left.10 \cdot \tau_{4 R}\right)$. This finding indicates that the reorientational dynamics of the investigated vectors cannot be described in terms of a
TABLE II. The calculated first, second, third, and fourth order Legendre reorientational correlation times for the $\mathrm{N}-\mathrm{H}$ and $\mathrm{N}-\mathrm{C}$ vectors of the $\mathrm{DMA}^{+}$cation.

\begin{tabular}{lcccc}
\hline \hline Vector & $\tau_{1 R}(\mathrm{ps})$ & $\tau_{2 R}(\mathrm{ps})$ & $\tau_{3 R}(\mathrm{ps})$ & $\tau_{4 R}(\mathrm{ps})$ \\
\hline $\mathrm{N}-\mathrm{H}$ & 10.8 & 4.5 & 1.7 & 0.9 \\
$\mathrm{~N}-\mathrm{C}$ & 9.6 & 2.8 & 1.4 & 0.7 \\
\hline \hline
\end{tabular}

diffusive model. The calculated reorientational correlation times for the $\mathrm{N}-\mathrm{H}$ and $\mathrm{N}-\mathrm{C}$ vectors of $\mathrm{DMA}^{+}$in bulk water are also larger than the corresponding values for the dipole moment vectors of its three nearest water neighbors, already presented above, indicating that the rotational motions of the cation are slightly slower.

The translational dynamics of the water molecules and the cation have been investigated in terms of the oxygen atom and the c.m. velocity TCFs, respectively,

$$
C_{v}{ }^{i}(t)=\frac{\left\langle\vec{v}_{i}(0) \cdot \vec{v}_{i}(t)\right\rangle}{\left\langle\vec{v}_{i}(0)^{2}\right\rangle} .
$$

The associated spectral densities, $S_{v}{ }^{i}(\omega)$, were also calculated by performing a Fourier transform,

$$
S_{v}{ }^{i}(\omega)=\int_{0}^{\infty} \cos (\omega \cdot t) \cdot C_{v}{ }^{i}(t) \cdot d t .
$$

Here, $S_{v}{ }^{i}(\omega)$ have been calculated by numerical integration using a Bode rule and also applying a Hanning window. In this way, the intermolecular vibrational dynamics and rattling dynamics of ions within a temporary cage made of neighboring solvent molecules can be studied in an efficient way. The calculated c.m. velocity TCFs and corresponding normalized $\left(S_{v}{ }^{i}(\omega) / S_{v}{ }^{i}(0)\right)$ spectral densities are presented in Figs. 11(a) and 11(b). From these data, it may be observed that the differences in the time decay of the calculated velocity TCFs depicted in Fig. 11(a) can be clearly reflected on the obtained spectral densities [Fig. 11(b)]. More specifically, the low frequency peak observed at $35 \mathrm{~cm}^{-1}$ in the case of the $\mathrm{DMA}^{+}$cation is shifted to higher frequencies in the case of water $\left(48 \mathrm{~cm}^{-1}\right)$. A shoulder and a small peak are also observed at around $250 \mathrm{~cm}^{-1}$ in both the water and $\mathrm{DMA}^{+}$spectral densities. The first low-frequency peak reflects more the cage effects, whereas the second band could be interpreted as more closely related to the mixture of underlying mechanisms, including hydrogen bridge bonds and cage effects.

The stronger $\mathrm{HB}$ interactions of the $-\mathrm{NH}_{2}$ group with the water molecules in comparison with the methyl ones are also reflected on the higher frequency part of the spectrum $\left(185 \mathrm{~cm}^{-1}-350 \mathrm{~cm}^{-1}\right)$ corresponding to the atomic velocity TCFs of the $\mathrm{H}_{\mathrm{N}}$ and the $\mathrm{H}_{\mathrm{M}}$ hydrogen atoms [Fig. 11(c)]. In that particular frequency range, several peaks are observed in the spectral density corresponding to the $\mathrm{H}_{\mathrm{N}}$ atoms, which are absent in the case of the $\mathrm{H}_{\mathrm{M}}$ hydrogen atoms. This is a clear indication of the existence of intermolecular vibrational motions due to the formation of $\mathrm{HB}$ dimers with the nearest water molecules. 

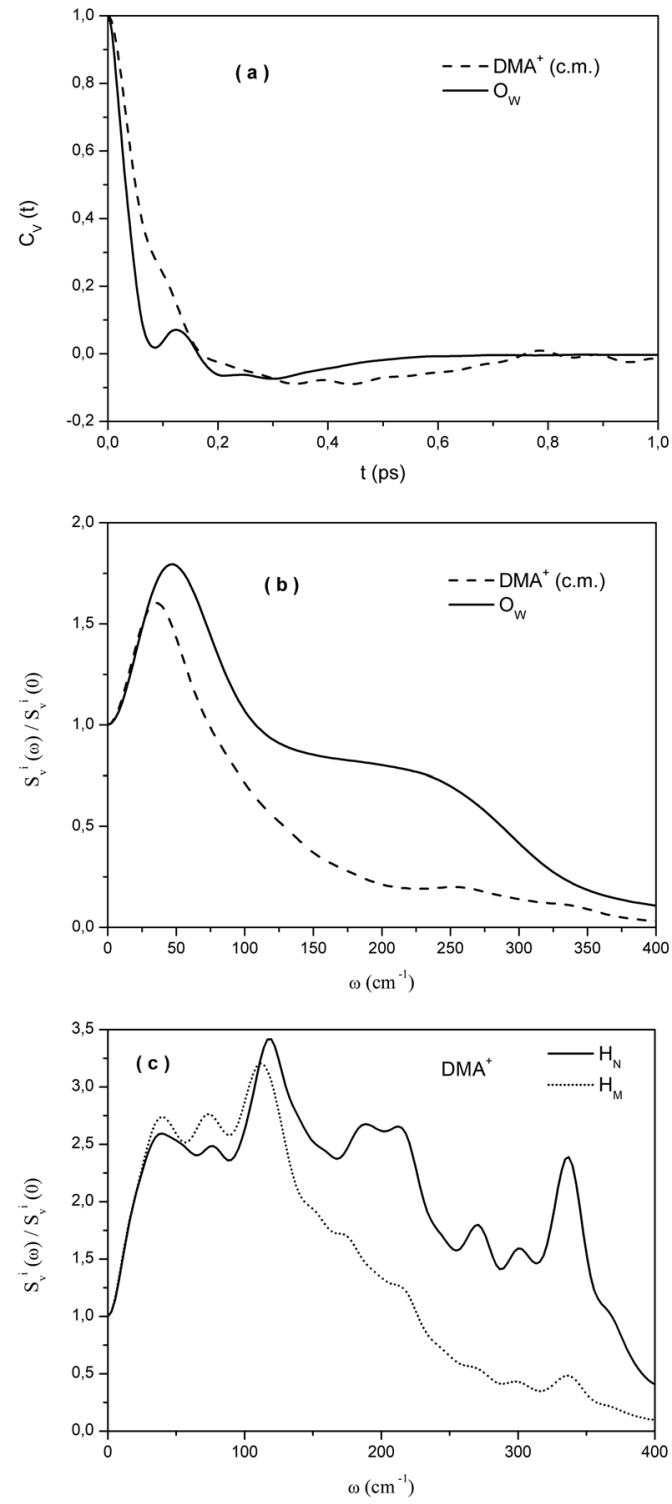

FIG. 11. Calculated (a) c.m. velocity TCFs and (b) corresponding normalized $\left[S_{v}{ }^{i}(\omega) / S_{V}{ }^{i}(0)\right]$ spectral densities. (c) Normalized $\left[S_{V}{ }^{i}(\omega) / S_{v}{ }^{i}(0)\right]$ spectral densities corresponding to the atomic velocity TCFs of the $\mathrm{H}_{N}$ and the $\mathrm{H}_{M}$ hydrogen atoms of the cation.

In order to provide more detailed information about the behavior of the water molecules inside the first solvation shell of the cation, the oxygen atomic velocity TCFs of the three nearest neighbors of the cation at time $\mathrm{t}=0$ and their corresponding spectral densities were also calculated, and they are presented in Fig. 12. As may be observed, the differences in the calculated velocity TCFs are clearly reflected on the behavior of the corresponding spectral densities in the frequency range from $150 \mathrm{~cm}^{-1}$ to $350 \mathrm{~cm}^{-1}$. In the case of the first water neighbor, the calculated spectral density exhibits a shoulder at $146 \mathrm{~cm}^{-1}$, followed by a peak at $255 \mathrm{~cm}^{-1}$ and
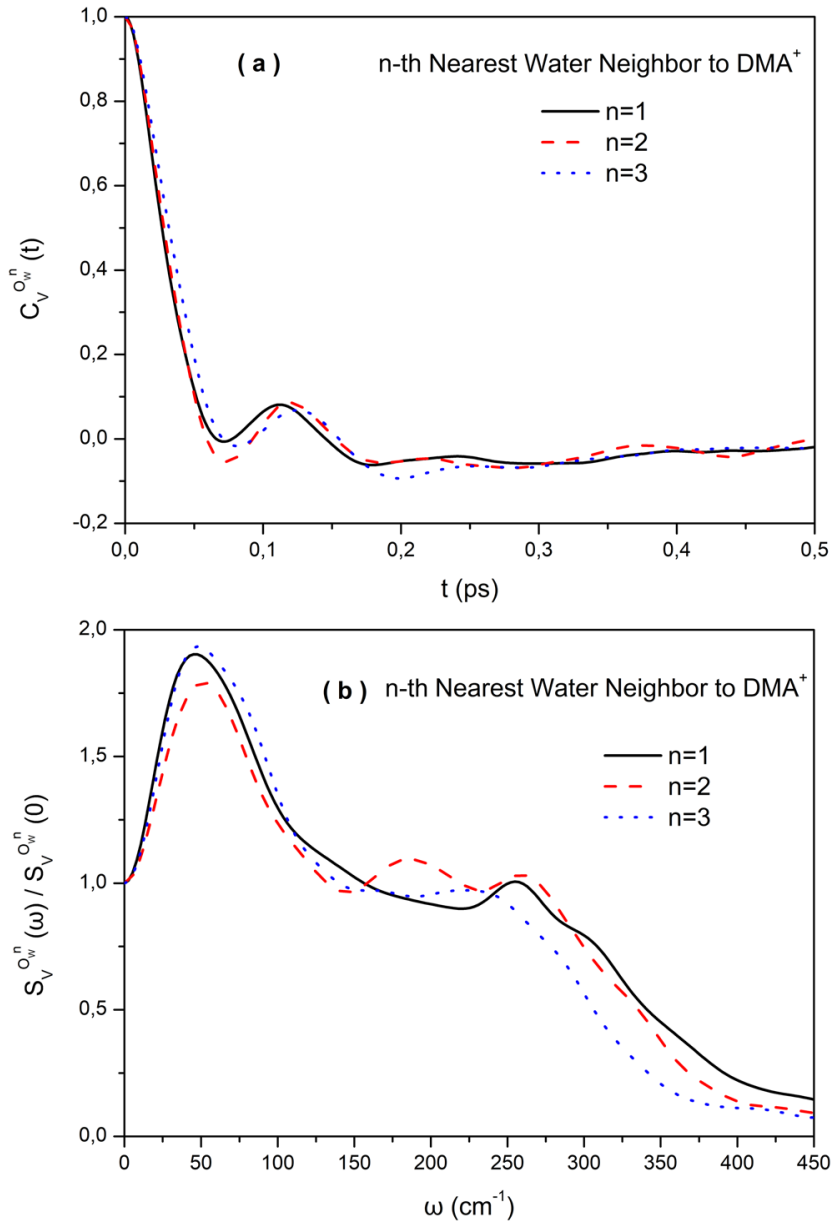

FIG. 12. Calculated (a) oxygen atomic velocity TCFs of the three nearest neighbors of the cation at time $\mathrm{t}=0$ and $(\mathrm{b})$ their corresponding normalized spectral densities $\left[S_{v}{ }^{i}(\omega) / S_{v}{ }^{i}(0)\right]$.

another shoulder at $325 \mathrm{~cm}^{-1}$. In the case of the second neighbor, two clear peaks are observed at $186 \mathrm{~cm}^{-1}$ and $260 \mathrm{~cm}^{-1}$, whereas the shoulder at about $325 \mathrm{~cm}^{-1}$ is much less pronounced. Finally, the spectral density corresponding to the third neighbor exhibits a shoulder at about $155 \mathrm{~cm}^{-1}$, followed by a weak peak at $222 \mathrm{~cm}^{-1}$. Taking into account our previous findings, revealing the preferential stronger $\mathrm{HB}$ interactions of the two nearest water neighbors with the $-\mathrm{NH}_{2}$ group of the cation, the higher frequency modes observed at about $325 \mathrm{~cm}^{-1}$ should be related to these particular $\mathrm{HB}$ interactions. On the other hand, the lower frequency modes in the range $147 \mathrm{~cm}^{-1}-186 \mathrm{~cm}^{-1}$ should be more closely related to the weaker interactions of the three nearest water molecules with the cation. The modes observed in the region $222 \mathrm{~cm}^{-1}$ $260 \mathrm{~cm}^{-1}$, which exhibit similarities with the behavior of the average spectral densities for all the water molecules, should be therefore more closely related to the interactions of hydrogen bonded water dimers. 
The translational mobility of the water molecules and the $\mathrm{DMA}^{+}$cation was also investigated by extracting the translational mean square displacements from the MD trajectories. These data were further used to calculate the translational self-diffusion coefficients of both species via the well-known Einstein relation,

$$
D_{S}=\frac{1}{6} \lim _{t \rightarrow \infty} \frac{1}{t}\left\langle\left|\vec{r}_{i}(0)-\vec{r}_{i}(t)\right|^{2}\right\rangle .
$$

The calculated self-diffusivities for $\mathrm{H}_{2} \mathrm{O}$ and $\mathrm{DMA}^{+}$are $D_{S}\left(\mathrm{H}_{2} \mathrm{O}\right)$ $=2.4 \cdot 10^{-9} \mathrm{~m}^{2} \mathrm{~s}^{-1}$ and $D_{S}\left(\mathrm{DMA}^{+}\right)=2.1 \cdot 10^{-9} \mathrm{~m}^{2} \mathrm{~s}^{-1}$, respectively. When comparing with the corresponding values under confinement in the Y-shp-MOF-5 nanoporous material, ${ }^{29}$ we may observe an order of magnitude increase in the diffusion coefficient of water and two orders of magnitude increase in the diffusion of the cation. Such a finding indicates that at the bulk solution, at dilute concentrations, the translational motions of the much larger cation are not as much restricted as under confinement, and they are mainly controlled by the translational mobility of the water molecules in the bulk liquid.

\section{CONCLUSIONS}

In the present treatment, classical molecular dynamics simulation techniques were employed to investigate the local solvation structure and related dynamics of the dimethylammonium cation diluted in liquid water at ambient conditions. The existence of two methyl groups and two hydrogen atoms in $\mathrm{DMA}^{+}$leads to the creation of a first solvation shell at short distances, which differs substantially from the $\mathrm{TMA}^{+}$case. ${ }^{30}$ On the other hand, the location of the second solvation shell is very similar in both cations, as well as the calculated coordination number. The average coordination number corresponding to the radius of the first solvation shell has been found to be 2.4. Our analysis revealed that the majority of the obtained first solvation shell configurations contain two water molecules, and there is a significant fraction of configurations containing three water molecules in the first solvation shell. It has also been revealed that the two nearest water neighbors of the $\mathrm{DMA}^{+}$cation approach the two $\mathrm{H}_{\mathrm{N}}$ atoms of the cation and form hydrogen bonds with them, whereas the third water neighbor interacts mainly with the methyl groups of the cation. The local hydration structure around $\mathrm{DMA}^{+}$is substantially different from the one recently reported ${ }^{31}$ for $\mathrm{NH}_{4}{ }^{+}$as well, with a first solvation shell located at $3.54 \AA$ formed by four tightly bound water molecules and a fifth water molecule that is exchanged between the first and second solvation shells. The recently reported hydration structure around $\mathrm{NH}_{4}{ }^{+}$was also found to be very ordered, and the four nearest water molecules that reside within the first solvation shell are hydrogen bonded with the hydrogen atoms of ammonium, which together form a distinct tetrahedral cage around the ammonium ion. The local orientational structure around the $\mathrm{DMA}^{+}$cation in the mixture was also investigated in terms of angular distributions and the trigonal order parameter. The results obtained have revealed a bimodal probability distribution function for the trigonal order parameter, indicating the existence of solvation structures with low and high trigonal symmetries of the water molecules around the cation. It has also been observed that the local orientational structure around the
$\mathrm{DMA}^{+}$cation affects strongly the local dielectric properties of its solvation shell, an effect which is clearly reflected on the behavior of the calculated local Kirkwood factor. All these findings indicate the very significant modifications in the hydration structure of $\mathrm{DMA}^{+}$, $\mathrm{TMA}^{+}$, and $\mathrm{NH}_{4}{ }^{+}$caused by the substitution of hydrogen atoms with methyl groups.

The calculated $\mathrm{HB}$, local orientational order, and reorientational dynamics have also revealed a significant speed-up of these relaxation phenomena in comparison with the corresponding $\mathrm{DMA}^{+}$aqueous solutions under confinement. Interestingly, a two-order of magnitude increase in the self-diffusion of the cation was observed in the bulk solution in comparison with the confined one. A corresponding one-order of magnitude increase was observed for the self-diffusion coefficient of water. The observation of very similar self-diffusion coefficients for the $\mathrm{DMA}^{+}$cation and the water molecules in the bulk dilute solution indicates that the translational motions of the much larger cation are not as much restricted as under confinement, and they are mainly controlled by the translational dynamics of the water molecules.

\section{ACKNOWLEDGMENTS}

I.S. acknowledges the use of the computational facilities of the Computer Simulation in Condensed Matter Research Group (SIMCON) at the Department of Physics of the Technical University of Catalonia (UPC). E.G. acknowledges financial support from the Ministerio de Ciencia, Innovación y Universidades of Spain, Grant No. PGC2018-099277-B-C21 (MCIU/AEI/ERDF).

\section{DATA AVAILABILITY}

The data that support the findings of this study are available from the corresponding author upon reasonable request.

\section{REFERENCES}

${ }^{1}$ Y. Marcus, Chem. Rev. 109, 1346 (2009).

${ }^{2}$ R. W. Gurney, Ionic Processes in Solution (McGraw-Hill, New York, 1953).

${ }^{3}$ E. Brini, C. J. Fennell, M. Fernandez-Serra, B. Hribar-Lee, M. Lukšič, and K. A. Dill, Chem. Rev. 117, 12385 (2017).

${ }^{4}$ H. Ohtaki and T. Radnai, Chem. Rev. 93, 1157 (1993).

${ }^{\mathbf{5}}$ B. Hribar, N. T. Southall, V. Vlachy, and K. A. Dill, J. Am. Chem. Soc. 124, 12302 (2002).

${ }^{6}$ O. Y. Samoilov, Discuss. Faraday Soc. 24, 141 (1957).

${ }^{7}$ M. Kondoh, Y. Ohshima, and M. Tsubouchi, Chem. Phys. Lett. 591, 317 (2014).

${ }^{8}$ G. A. Krestov, Thermodynamics of Solvation (Ellis Harwood, New York, 1990).

${ }^{9}$ J. Duboisset and P.-F. Brevet, Phys. Rev. Lett. 120, 263001 (2018).

${ }^{10}$ K. A. Dill, T. M. Truskett, V. Vlachy, and B. Hribar-Lee, Annu. Rev. Biophys. Biomol. Struct. 34, 173-199 (2005).

${ }^{11}$ K. D. Collins, Biophys. J. 72, 65 (1997).

${ }^{12}$ R. Mancinelli, A. Botti, F. Bruni, M. A. Ricci, and A. K. Soper, Phys. Chem. Chem. Phys. 9, 2959 (2007).

${ }^{13}$ E. Guàrdia, I. Skarmoutsos, and M. Masia, J. Chem. Theory Comput. 5, 1449 (2009).

${ }^{14}$ E. Guàrdia, D. Laria, and J. Martí, J. Phys. Chem. B 110, 6332 (2006).

${ }^{15}$ S. Koneshan, J. C. Rasaiah, R. M. Lynden-Bell, and S. H. Lee, J. Phys. Chem. B 102, 4193 (1998).

${ }^{16}$ G. Stirnemann, E. Wernersson, P. Jungwirth, and D. Laage, J. Am. Chem. Soc. 135, 11824 (2013). 
${ }^{17}$ C. D. Wick and S. S. Xantheas, J. Phys. Chem. B 113, 4141 (2009).

${ }^{18}$ L. Suo, O. Borodin, T. Gao, M. Olguin, J. Ho, X. Fan, C. Luo, C. Wang, and K. Xu, Science 350, 938 (2015).

${ }^{19}$ Z. Li, R. Bouchal, T. Mendez-Morales, A.-L. Rollet, C. Rizzi, S. Le Vot, F. Favier, B. Rotenberg, O. Borodin, O. Fontaine, and M. Salanne, J. Phys. Chem. B 123, 10514 (2019).

${ }^{20}$ S. Chowdhuri and A. Chandra, J. Chem. Phys. 115, 3732 (2001).

${ }^{21}$ Y. Shinohara, R. Matsumoto, M. W. Thompson, C. W. Ryu, W. Dmowski, T. Iwashita, D. Ishikawa, A. Q. R. Baron, P. T. Cummings, and T. Egami, J. Phys. Chem. Lett. 10, 7119 (2019).

${ }^{22}$ S. Roy, S. Yashonath, and B. Bagchi, J. Chem. Phys. 142, 124502 (2015).

${ }^{23}$ J. Chandrasekhar and W. L. Jorgensen, J. Chem. Phys. 77, 5080 (1982).

${ }^{24}$ G. Balasubramanian, S. Murad, R. Kappiyoor, and I. K. Puri, Chem. Phys. Lett. 508, 38 (2011).

${ }^{25}$ V. Migliorati, A. Serva, F. Sessa, A. Lapi, and P. D'Angelo, J. Phys. Chem. B 122, 2779 (2018)

${ }^{26}$ J. Teychené, H. R.-d. Balmann, L. Maron, and S. Galier, J. Mol. Liq. 294, 111394 (2019).

${ }^{27}$ W. Rudolph, M. H. Brooker, and C. C. Pye, J. Phys. Chem. 99, 3793 (1995).

${ }^{28}$ P. I. Nagy, Phys. Chem. Chem. Phys. 14, 849 (2012).

${ }^{29}$ I. Skarmoutsos, M. Eddaoudi, and G. Maurin, J. Phys. Chem. C 123, 26989 (2019).

${ }^{30}$ L. García-Tarrés and E. Guàrdia, J. Phys. Chem. B 102, 7448 (1998).

${ }^{31}$ F. Aydin, C. Zhan, C. Ritt, R. Epsztein, M. Elimelech, E. Schwegler, and T. A. Pham, Phys. Chem. Chem. Phys. 22, 2540 (2020).

${ }^{32}$ L. Martínez, R. Andrade, E. G. Birgin, and J. M. Martínez, J. Comput. Chem. 30, 2157 (2009)

${ }^{33}$ A. V. Onufriev and S. Izadi, Wiley Interdiscip. Rev.: Comput. Mol. Sci. 8, e1347 (2018).

${ }^{34}$ A. V. Onufriev and S. Izadi, J. Chem. Phys. 145, 074501 (2016).

${ }^{35}$ H. J. C. Berendsen, J. R. Grigera, and T. P. Straatsma, J. Phys. Chem. 91, 6269 (1987).
${ }^{36}$ I. Skarmoutsos, E. Guardia, and J. Samios, J. Supercrit. Fluids 130, 156 (2017).

${ }^{37}$ Y. Guissani and B. Guillot, J. Chem. Phys. 98, 8221 (1993).

${ }^{38}$ E. Guardia and J. Marti, Phys. Rev. E 69, 011502 (2004).

${ }^{39}$ I. Skarmoutsos, L. Spyrogiannopoulos, E. Kainourgiakis, and J. Samios, J. Mol. Liq. 289, 111135 (2019).

${ }^{40}$ M. P. Allen and D. J. Tildesley, Computer Simulations of Liquids (Oxford University Press, Oxford, 1987).

${ }^{41}$ W. G. Hoover, Phys. Rev. A 31, 1695 (1985).

${ }^{42}$ W. G. Hoover, Phys. Rev. A 34, 2499 (1986).

${ }^{43}$ W. Smith and T. R. Forester, J. Mol. Graphics 14, 136 (1996).

${ }^{44}$ A. Idrissi, P. Damay, and M. Kiselev, Chem. Phys. 332, 139 (2007).

${ }^{45}$ I. Skarmoutsos, S. Mossa, and J. Samios, J. Chem. Phys. 145, 154505 (2016).

${ }^{46}$ I. Skarmoutsos, S. Mossa, and E. Guardia, J. Chem. Phys. 150, 124506 (2019).

${ }^{47}$ H. Tanaka, H. Tong, R. Shi, and J. Russo, Nat. Rev. Phys. 1, 333 (2019).

${ }^{48}$ R. H. Henchman and S. J. Cockram, Faraday Discuss. 167, 529 (2013).

${ }^{49}$ I. Skarmoutsos, M. Masia, and E. Guardia, Chem. Phys. Lett. 648, 102 (2016).

${ }^{50}$ I. Skarmoutsos, V. Ponnuchamy, V. Vetere, and S. Mossa, J. Phys. Chem. C 119, 4502 (2015).

${ }^{51}$ M. Neumann, Mol. Phys. 50, 841 (1983).

${ }^{52}$ J. Anderson, J. J. Ullo, and S. Yip, J. Chem. Phys. 87, 1726 (1987).

${ }^{53}$ S. Peng, Q. Zeng, X. Yang, J. Hu, X. Qiu, and J. He, Sci. Rep. 6, 38978 (2016).

${ }^{54}$ D. C. Elton, Phys. Chem. Chem. Phys. 19, 18739 (2017).

${ }^{55}$ D. C. Rapaport, Mol. Phys. 50, 1151 (1983).

${ }^{56}$ I. Skarmoutsos and E. Guardia, J. Phys. Chem. B 113, 8887 (2009).

${ }^{57}$ A. Luzar and D. Chandler, Nature 379, 55 (1996).

${ }^{58}$ E. Guardia, I. Skarmoutsos, and M. Masia, J. Phys. Chem. B 119, 8926 (2015).

${ }^{59}$ P. Debye, Polar Molecules (Dover Publications, New York, 1929).

${ }^{60}$ B. J. Berne and R. Pecora, Dynamic Light Scattering: With Applications to Chemistry, Biology and Physics (John Wiley \& Sons, New York, 1976).

${ }^{61}$ D. Laage and J. T. Hynes, J. Phys. Chem. B 112, 14230 (2008).

${ }^{62} \mathrm{M}$. Galvin and D. Zerulla, ChemPhysChem 12, 913 (2011). 